\title{
History of Telemedicine: Evolution, Context, and Transformation
}

\section{Seewon Ryu, PhD}

Department of Public Health and Healthcare Management, Inje Institute of Advanced Studies seewon@inje.ac.kr

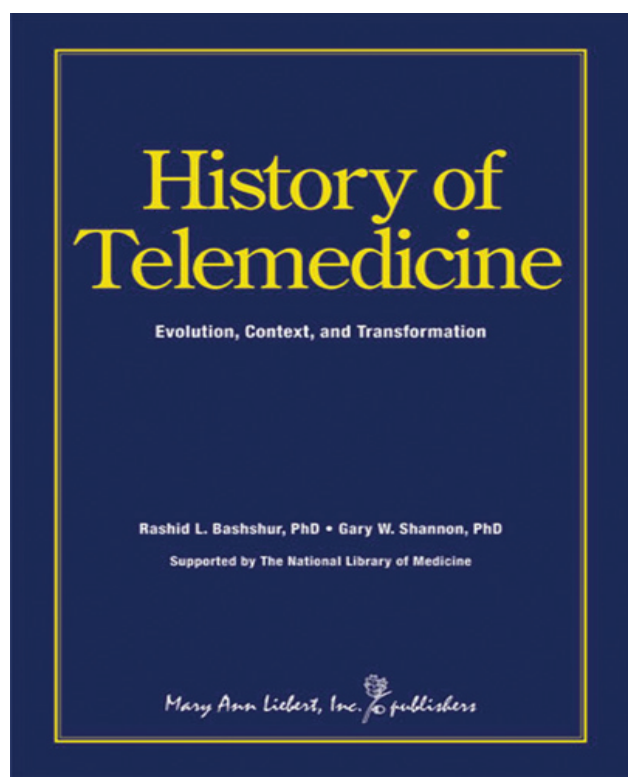

By Rashid L. Bashshur, PhD, and Gary W. Shannon, PhD New Rochelle (NY): Mary Ann Liebert, Inc.; 2009. ISBN 13 978-1-934854-11-2 • ISBN e-book 1-934854-04-2 Hardcover $\bullet 415$ pages, 107 illus $\bullet$ Trim size: $7 \times 10$ $\$ 195$ US • \$215 Outside US • \$195 e-book Personal • \$585 e-book Inst site-license

This is an Open Access article distributed under the terms of the Creative Commons Attribution Non-Commercial License (http://creativecommons.org/licenses/by$\mathrm{nc} / 3.0 /$ ) which permits unrestricted non-commercial use, distribution, and reproduction in any medium, provided the original work is properly cited.

(c) 2010 The Korean Society of Medical Informatics
We are living in the age of high information and communication technology, and have long-standing problem of improving human health in healthcare systems. The History of Telemedicine provides a comprehensive and in-depth historical view of telemedicine from ancient Greece to the present time. The authors started the task of writing the book with open mind and put aside whatever preconceived notion or information about telemedicine. Bashshur and Shannon really give historical insight to us on telemedicine. It would be a good guide and rationale for the telemedicine.

Before authors began to trace and document the history of telemedicine, they reviewed the published literature. They clearly realized the storytelling of telemedicine, because telemedicine has a long and rich history and a story that has not been told, certainly not in its entirety. Two common characteristics were revealed after careful review of the history of telemedicine in the published literature. First, they tend to be very brief, often cursory or selective, sometimes limited to a couple of paragraphs, and are typically included as a prelude to another topic. Second, they are invariably simple accounts of early attempts to connect providers and patients through telecommunication devices available at that time. They wanted to be guided solely by the documented not by any preexisting ideas or notions, and therefore they were to minimize bias in reporting.

The journey started with ancient societies and the early attempts to establish rudimentary communication connectivity between settlements when faced with internal or external threats and subsequently to establish clinical connectivity between patient and physician/caregiver/priest. Thus, throughout this book, authors emphasized the continuing underlying theme of connectivity between those in need of care and those who provide it. Authors traced the history of long-distance communication from its humble origins 
in semaphore, and much later the telegraph, and radio to advanced digital communication and computer processing systems. Telemedicine provided the tools for connectivity when providers and recipients of care could not be in the same place and time.

Authors wanted to achieve some measures of success in contributing to the full story of telemedicine, the evolution of pertinent information and communication technology, and the broader healthcare context, dating back to ancient societies and continuing through to the present. They traced the origin of modern telemedicine applications in Europe and the fact that those by a Dutch physician, Willem Einthoven, had been by long distance transfer of electrocardiograms in 1905. Authors, after careful scrutiny of various sources, can attest that the first clinical application in telemedicine was in cardiology, not radiology as some might have contended. This was followed by radio consultations from medical centers in Norway, Italy, and France in the 1920s, 1930s, and 1940s for patients aboard ships at sea and on remote islands. The transmission of radiographic images began in the early 1950s in the United States, followed shortly thereafter by similar experimentation in Canada. The first wave of organized telemedicine programs in the United States began in the late 1950s. It lasted nearly two decades and then came to a halt shortly after extramural funding was terminated. This was followed by a hiatus that lasted nearly a decade, until a new wave of telemedicine projects and programs developed at a much larger scale than its forerunner. This last wave was led by state-based and province-based initiatives throughout the United States and Canada.

The search also led to some unexpected findings that may dispel other claims perpetuated through verbal communication and presentations at professional meetings without adequate verification. The deep roots of telemedicine are available in the archives of specialty areas in medicine (such as cardiology and radiology) and telecommunications and electronics is not necessarily available in the literature of telemedicine per se.

In probing the history of telemedicine from the ancient to the present time, authors discovered continuity and change existing side by side in a dynamic evolutionary process. Continuity stems from the convergence of medical care delivery and distance communication in various forms and manifestations, whereas change reflects the never-ending advances in the character and capability of the technology that enables telemedicine as well as other concurrent advances in medical science and medical practice.

The major perspective of the book is one of evolution, context, and transformation in the history of telemedicine. However, authors paid special attention to the broader context for the development of telemedicine, which also explains its endurance despite cycles of advancement and retrenchment, of peaks and valleys in its long history from the ancient societies. Especially, they were to see through the history of telemedicine by a reasonable understanding of the healthcare context for its development and its persistence through numerous decades.

The book does not only review the comprehensive history of telemedicine, but also discuss the telemedicine solution and proposed an assessing tool for it. It is the matrix for assessing telemedicine, by which we can evaluate the benefits of telemedicine modality in three perspectives: those of the providers, the clients or patients, and society at large.

Nowadays, the nomenclature of telemedicine is created newly as "u-Health" in which ubiquitous technology is using to connect provider and patients in ubiquity. Technological system of $\mathrm{u}$-Health will be easily established by convergence among ubiquitous and biometric technology, and mobile telecommunication infrastructure. However, the crucial point of the adoption and success of telemedicine in even ubiquity environments needs enough to tightly harmonize with the mainstream medicine.

The History of Telemedicine would give invaluable and experiential lessons to the present and future direction of it. The book, reviewing about why and way of telemedicine from the past to the present, provides to the stakeholders of telemedicine an interesting and insightful perspective. Although not completely comprehensive review telemedicine experiences in the world, it provides many lessons and thoughtful chances to the reader. It is recommended for not only those who have special interests on telemedicine, but also those who are searching for new way of solving the current pentup demand or unmet need. 